\title{
IMPETIGO CONTAGIOSA
}

\author{
RESULTS CF CULTURES MADE OF THIRTY CASES WITH SPECIAL REFERENCE \\ TO THE CHARACTER OF STREPTOCOCCI ISOLATED *
}

DAVID L. FARLEY, M.D., AND FRANK CROZER KNOWLES, M.D. PHILADELPHIA

\section{BACTERIOLOGIC REVIEW}

Impetigo (contagiosa) was definitely described as a clinical entity in 1864 and 1869 by Tilbury Fox. ${ }^{1}$ Kohn, ${ }^{2}$ in 1871, concluded that it was due to an organism of the hyphomycete group. In this he was supported by Behrend, ${ }^{3}$ but this view has been generally abandoned in recent years. Demme, ${ }^{4}$ in 1886 , was the first to cultivate a diplococcus from a case of acute contagious pemphigus. Bockhart, ${ }^{5}$ in 1887, concluded that impetigo was caused by Staphylococcus pyogenes aureus and albus.

In 1893, M. C. Leroux ${ }^{6}$ took cultures from 120 children with cases of contagious impetigo. He obtained seventy positive cultures, finding divers varieties of Staphylococcus pyogenes and streptococci. He called attention to the contagious character of the disease and its appearance among children in the same family and after contact. Studying impetigo experimentally, he inoculated a series of children, reproduced typical lesions, and on reculture of the lesions found diplococci and the typical streptococci-but no staphylococci. He concluded that impetigo was caused by a special organism to which he gave the name "streptococcus of impetigo." He concluded that the staphylococci were superimposed organisms which rapidly substituted the streptococci and produced by secondary infection the several clinical manifestations. Davalos, ${ }^{7}$ in 1895 , gave as the exciting cause a pseudodiphtheria bacillus in symbiosis with a virulent coccus. This view has received little encouragement from later investigators.

\footnotetext{
* From the Laboratory of Dermatological Research, Department of Cutaneous Medicine, University of Pennsylvania and the Department of Dermatology, Jefferson Medical College.

1. Fox, Tilbury: Brit. M. J., 1864, pp. 467, 495, 553, 607; J. Cutan. Med. 3: 231 (Oct.) 1869.

2. Kohn, M.: Wien. med. Presse, 1871, p. 586.

3. Behrend, H.: Deutsch. med. Wchnschr., No. 48, 1884.

4. Demme, Bern: Verhandl. Cong. f. inn. Med. W., 1886, p. 336.

5. Bockhart, Max: Monatschr. f. prakt. Dermat., No. 11, 1887, p. 450.

6. Leroux, C.: Ann. de dermat. et syph., 1893, No. 4, p. 290.

7. Davalos, J. N.: Zentralbl. f. Bakteriol. 17:38, 1895.
} 
Balzer and Griffon ${ }^{8}$ have analyzed fourteen cases of ecthyma and thirty-one cases of impetigo bacteriologically. They found the streptococcus in all. It was present in pure culture in the pus of unopened pustules as a diplococcus or as chains. On culture the organism showed all the classic characteristics of Streptococcus pyogenes. Injected into animals it caused erysipelas, abscess and fatal septicemia. Matzenauer ${ }^{9}$ concluded, in 1900, that impetigo contagiosa and pemphigus neonatorum were identical and due to a staphylococcus.

In reference to pemphigus neonatorum it may be proper at this point to review the recent work of Cole and Ruh, ${ }^{10}$ and Falls. ${ }^{11}$

Cole and Ruh, in an epidemic of nine cases of infantile pemphigoid (pemphigus neonatorum), isolated Staphylococcus aureus in pure culture in all cases in which the vesicles were unbroken. The epidemic started as a case of typical pemphigoid of the new-born which later changed into a clinical picture of dermatitis exfoliativa neonatorum; and as the etiologic agent was thus the same, they believed there should be no distinction between the two conditions. Impetigo contagiosa, they say, should be sharply differentiated from this infantile pemphigoid (pemphigus neonatorum) because of its different bacteriologic origin. They believed pemphigus neonatorum should be made reportable on account of its high mortality ( 25 to 50 per cent). They recommended autogenous vaccine.

Falls reported fifty-four cases of pemphigus neonatorum from the standpoint of bacteriology, but did not state from how many cultures were made. He concluded that Staphylococcus aureus was the cause.

In 1900, Sabouraud ${ }^{12}$ divided impetigo cases into two main divisions-the vesicular type of Tilbury Fox and the pustular type of Bockhart-the first primarily due to a streptococcus which, as a rule, became secondarily associated with staphylococci after which the streptococci died out.

Gilchrist ${ }^{13}$ has reported the results of cultivation in seventeen cases. Streptococcus pyogenes was obtained from every case, in ten cases in pure culture. In seven cases Staphylococcus aureus was also present, in one case Staphylococcus citreus and albus, and in another instance a pseudodiphtheria bacillus was present in addition to a streptococcus. Streptococci from only three cases were run through mediums and

8. Balzer and Griffon: Presse méd., Oct. 27, 1897, No. 89, p. 130.

9. Matzenauer, R.: Virchow-Hirsch. Jahrb. d. ges. Med. 25:549, 1900.

10. Cole, H. N., and Ruh, H. O.: Pemphigoid of the New-Born (Pemphigus Neonatorum), J. A. M. A. 63:1159 (Oct. 3) 1914.

11. Falls, F. H.: J. Infect. Dis. 20:86 (Jan.) 1917.

12. Sabouraud, R.: Ann. de dermat. et syph. 31:325, 1900.

13. Gilchrist, T. C.: Contribution to Science of Medicine by Pupils of W. H. Welch, 1900, p. 409. 
identified definitely as Streptococcus pyogenes. In one instance he obtained a typical lesion by inoculating the chin with a pure culture of streptococci obtained from the same patient. This outbreak developed five days after the inoculation. These results agree in general with those obtained by Leroux, Balzer and Griffon, and Sabouraud. He concluded that his investigations seemed to indicate that impetigo contagiosa was caused by Streptococcus pyogenes.

Bender ${ }^{14}$ concluded from cultural studies of twelve cases in 1907 that the cause of impetigo contagiosa was not the white staphylococcus but rather a streptococcus which appeared in chains and gave the typical medium reactions of a streptococcus.

According to Ernst Flehme: ${ }^{15}$

In fifty-five cases of impetigo contagiosa investigated bacteriologically, streptococci in pure culture or mixed with staphylococci, according to the age of the lesion, were found. In every case, the organism was of the Streptococcus longus type. The organisms always showed hemolysis and acid formation on mannite-litmus-agar. The impetigo streptococci were pathogenic for white mice when large doses of the sediment of bouillon cultures were injected. At the site of injection, a circumscribed purulent lesion was formed. In man it was possible through superficial inoculation to form typical lesions of impetigo, from which the organism was recovered in pure culture. In 15 per cent. of the skins of normal persons, and in 89 per cent. of persons with impetigo, it was possible to demonstrate streptococci which could only be differentiated from the impetigo streptococci by a stronger red tint of mannite-litmus-agar, and hemolysis. The superficial inoculation of pure cultures of streptococci recovered from normal skin likewise produced an impetigo. In addition, scarification of healthy skin on which streptococci were demonstrable, led to the formation of impetigo. ${ }^{16}$

His conclusions were that:

. . . the cause of impetigo is the attack on fertile ground of a large dose of impetigo streptococci. The normal skin appears to have a resistance to the organism. The skin of a patient with impetigo has a lessened resistance to the same organism, yet there remains some resistance. One may consider the impetigo streptococcus as a semiparasitic organism, that under certain circumstances and in certain localities, and with a diminished resistance of the skin, leads to the formation of the disease. In addition, during epidemics there may be an increased virulence of the organism which overcomes the normal resistance of the healthy skin.

\section{AUTHORS' WORK}

It will be seen from the foregoing that investigators of impetigo have gone no farther than to conclude that a streptococcus was causative or that a streptococcus was the organism most frequently and con-

14. Bender, E.: Arch. f. Dermat. u. Syph. 84:59, 1907.

15. Flehme, E.: Dermat. Ztschr. 31:111, 1920; abstr. Arch. Dermat. \& Syph. 2:761, 1920.

16. Quoted from abst., Arch. Dermat. \& Syph. 2:761 (Dec.) 1920. 
sistently isolated from cases of impetigo. It is well known that the streptococcus group is a large one and varies greatly in pathogenicity and biologic characteristics, embracing such widely differing organisms as the almost entirely parasitic pneumococcus on the one hand and the semisaprophytic Streptococcus fecalis on the other. We have attempted to go further, that is, we have "analyzed" the different streptococci of impetigo, and have classified them as completely as possible in order to determine, if possible, whether the organisms thus obtained belonged to a single specific strain or whether different members of the streptococcus group were concerned. It occurred to us that the somewhat varying clinical pictures of impetigo might be connected with different strains of streptococcus-just as the markedly impetiginous one of the muddy Flanders battlefields was characterized by heavy Streptococcus fecalis incidence.

\section{TECHNIC}

We have made cultures from thirty cases of impetigo, all showing lesions clinically typical of this disease. When possible the cultures were made from the serum of unbroken lesions. If not possible, the lesion was scraped with sterile swabs until bloody serum was obtained. In those lesions which had broken and become crusted it was found that cultures were more often positive for streptococci when the swab was taken as deeply as possible. This was similar to the experience of the French bacteriologists with their cultures from wounds; they found that a superficial swab from a wound often resulted in a culture of staphylococci only and the missing of streptococci when they were present.

Swabs were cultivated immediately on 5 per cent. human bloodagar plates, the agar being 0.5 per cent. acid to phenolphthalein and made from fresh beef. After twenty-four hours' inoculation organisms present were picked and seeded for pure culture. Streptococci were repeated on blood agar and picked a second time to insure purity before running them through the carbohydrate mediums for classification. All sugar mediums were made up in bouillon (titrated 0.5 per cent.), to which was added blood serum and Andrade indicator according to the method outlined by Holman. ${ }^{17}$

We consider the use of liquid mediums especially important in the identification of the streptococcus group, and agree with the many observers who have stressed the point that solid carbohydrate medium with litmus as an indicator is entirely unsatisfactory for precise work. We attempted the use of solid medium with litmus on several strains and discarded the results, the main difficulty being that it was often

17. Holman, W. L.: Jour. Infect. Dis. 15:209, 1914; ibid. 34:377, 1916. 
impossible to get a satisfactory growth, and it was often doubtful whether there was growth. Delicate color changes were difficult to interpret on solid litmus mediums with stab inoculations.

Along a different line we attempted to produce immune serum in rabbits for agglutination tests. We succeeded in obtaining antiserum which would agglutinate the strain used for inoculation (homologous antigen) in dilutions of 1:100, which was not satisfactorily high. Several strains of streptococci isolated were in a constant state of autoagglutination, which was impossible to break up even by shaking with sand, and the results on these strains were of course unreadable. The general results of agglutination tests with immune serum agreed with the carbohydrate differentiation tests, that is, the several strains of streptococci which had similar carbohydrate reaction to the strain used for production of the serum were cross-agglutinated, while those with different carbohydrate reactions were not agglutinated. However, the auto-agglutination of several strains and the low agglutination titer ( $1: 100)$ of serum produced make us hesitate to draw any definite conclusions as to specificity on the basis of our serologic reactions.

We have made no attempt to fulfil all of Koch's postulates, our animal experimentation work having been confined to the production of agglutinins in rabbits.

\section{BACTERIOLOGIC FINDINGS}

The bacteriologic findings in the thirty cases examined were: Streptococci were found in 24 cases (pure cultures in 10 cases); Streptococcus pyogenes 10, Streptococcus anginosis 11, Streptococcus subacidus 2, Streptococcus fecalis 1. Staphylococci were found 22 times in 16 cases (mostly associated with other organisms); Staphylococcus albus 16 times (2 pure), Staphylococcus aureus 6 times (never pure). Pseudodiphtheria bacillus was found 4 times, never pure. Bacillus pyocyaneous was found once and Staphylococcus citreus once.

As to the matter of simple streptococcic incidence, the foregoing data confirm the results of many other investigators who found that a streptococcus was almost invariably present in the lesions of impetigo. This and the successful inoculation tests of several workers convince us that the streptococcus is the causative organism. The staphylococci, pseudodiphtheria bacilli, etc., are secondary invaders.

From the standpoint of the different strains of streptococci, the data show that there are at least four concerned, and the probability is that on examination of a larger series of cases additional ones would appear. Streptococcus anginosus and Streptococcus pyogenes appear to be most numerous (and equally) important. We have followed the classification of Holman in our work. It will be noted that all the 
streptococci which we found were hemolytic save one (Streptococcus fecalis), and this was encountered only once.

We have plotted out the various combinations of the different bacterial groups (hemolyzers, staphylococci, pseudodiphtheria), but there does not appear to be anything consistent or suggestive in the combinations to indicate anything of practical value.

In the six cases failing to give streptococci in culture the lesions were of long duration.

\section{CONCLUSIONS}

With proper precautions and under proper conditions streptococci may be obtained from nearly all cases of impetigo. If the full complement of streptococci is to be obtained on culture, it is necessary to take the material from an early and unerupted lesion. If this is not possible, it should be taken from the deep, redder parts from which all crusts have been removed.

Accepting as we do the conclusions of previous workers that impetigo is caused by streptococci, at least four different strains are concerned: Streptococcus pyogenes, Streptococcus anginosus, Streptococcus subacidus and Streptococcus fecalis.

Associated organisms, such as staphylococci, pseudodiphtheria bacilli, etc., are either extraneous or secondary invaders.

Streptococcus fecalis is not the factor in America that it was in Flanders, and there is little likelihood that it has been recently imported.

It cannot be predicted from the aspect of the clinical lesion (bullous, crusted or ecthymatous) what strain of streptococcus will be found on culture. 